\title{
CONSTRUCTION OF OPERATORS WITH PRESCRIBED ORBITS IN FRÉCHET SPACES WITH A CONTINUOUS NORM
}

\author{
ANGELA A. ALBANESE
}

\begin{abstract}
Let $X$ be a separable, infinite dimensional real or complex Fréchet space admitting a continuous norm. Let $\left\{v_{n}: n \geq 1\right\}$ be a dense set of linearly independent vectors of $X$. We show that there exists a continuous linear operator $T$ on $X$ such that the orbit of $v_{1}$ under $T$ is exactly the set $\left\{v_{n}: n \geq 1\right\}$. Thus, we extend a result of Grivaux for Banach spaces to the setting of non-normable Fréchet spaces with a continuous norm. We also provide some consequences of the main result.
\end{abstract}

\section{Introduction}

Let $X$ be a separable, infinite dimensional Fréchet space over the scalar field $\mathrm{K}$, where $\mathrm{K}$ denotes either the real field $\mathrm{R}$ or the complex field C. Let $\mathscr{L}(X)$ denote the space of all continuous linear operators from $X$ into itself. Then an operator $T \in \mathscr{L}(X)$ is called hypercyclic if there exists a vector $x \in X$ such that the orbit of $x$ under $T$, that is, $\operatorname{Orb}(T, x)=\left\{x, T(x), T^{2}(x), \ldots\right\}$, is dense in $X$. Such a vector $x$ is called a hypercyclic vector for $T$.

Rolewicz [17] was the first to study hypercyclicity of operators in classical Banach spaces. He showed that no finite dimensional linear vector space supports a hypercyclic operator, and that if $B$ denotes the backward shift, i.e., $B\left(x_{n}\right)_{n}=\left(x_{n+1}\right)_{n}$, then for any $a>1$ the operator $T=a B$ is hypercyclic on $\ell^{p}, 1 \leq p<\infty$, and $c_{0}$, and for any $a>0$ it is hypercyclic on the space $\omega=\mathrm{K}^{\mathrm{N}}$ of all scalar sequences. He also asked in [17] whether any separable, infinite dimensional Banach space supports a hypercyclic operator. This question was solved in the affirmative, independently, by Ansari [1] and Bernal [2] for Banach spaces. This result was also extended to the non-normable Fréchet case by Bonet and Peris in [6]. The proofs of [1], [2] and [6] rely on a result of Salas [18, Theorem 3.3], who completely characterized the hypercyclic weighted shift operators on $\ell^{p}, 1 \leq p<\infty$, and $c_{0}$. Hypercyclic operators have been intensely studied during last years, the research starting with the investigations of Godefroy and Shapiro [9]; see the survey papers [5], [11], [12] and the references therein.

Received 2 February 2010, in final form 23 July 2010. 
Solving a problem of Halperin, Kitai and Rosenthal [13], Grivaux [10, Theorem 3.1] showed that if $\left\{v_{n}: n \geq 1\right\}$ is any countable set of linearly independent vectors in a separable, infinite dimensional Banach space $X$, then there exists an operator $T \in \mathscr{L}(X)$ such that $\operatorname{Orb}\left(T, v_{1}\right)$ contains the set $\left\{v_{n}: n \geq 1\right\}$. This result was proved in [13] in the case $X$ is a Hilbert space. Her proof relies on the existence result of hypercyclic operators given in [1], and on a deep technical lemma, [10, Lemma 2.1], concerning the existence of a topological isomorphism between any two dense sets of linearly independent vectors in separable, infinite dimensional Banach spaces. She also provided in such a paper some interesting consequences of [10, Lemma 2.1, Theorem 3.1]. For instance, she showed that any dense infinite dimensional linear subspace $M$ of countable dimension of a separable, infinite dimensional Banach space $X$ can be written as $M=\mathrm{K}[T](x)$, i.e., $M=\{p(T)(x): p \in \mathrm{K}[X]\}$, for some hypercyclic operator $T \in \mathscr{L}(X)$ and some hypercyclic vector $x \in M$. We recall the following well-known result: if $X$ is a separable, infinite dimensional Banach space over $\mathrm{K}, T \in \mathscr{L}(X)$ is a hypercyclic operator and $x$ is any hypercyclic vector for $T$, then $\mathrm{K}[T](x)$ is a dense invariant hypercyclic linear subspace for $T$, i.e., every non-zero vector of $\mathrm{K}[T](x)$ is hypercyclic for $T$, see the works of Bourdon [7], Herrero [14], Bès [3] and Wengenroth [19]. Thus, she obtained that every normed space of countable dimension supports an operator which has no non-trivial invariant closed set. This result is related to the "Invariant Set Problem". In contrast to the results of Grivaux, among other things Bonet, Frerick, Peris and Wengenroth showed in [4, Proposition 3.3] that neither Grivaux's main result [10, Theorem 3.1] nor the technical [10, Lemma 2.1] holds for non-normable Fréchet spaces. More precisely, they proved that there exists a dense linearly independent sequence in the Fréchet space $\omega$ of all complex sequences that cannot be the orbit of a hypercyclic operator on $\omega$, and that every countable product of copies of a separable, infinite dimensional Banach space $X$ contains two dense linearly independent sequences of vectors such that their linear spans are not isomorphic. They also provided an example of a countable dimensional locally convex space admitting no transitive operator (and hence, no hypercyclic operators), [4, Proposition 3.2].

We observe that $\omega$ and every countable product of copies of an infinite dimensional Banach space $X$ are all examples of non-normable Fréchet spaces no admitting a continuous norm. So, it is natural to consider the following question: do the Banach results mentioned above carry over to the setting of non-normable Fréchet spaces which admit a continuous norm?

The aim of this note is to show that all the Banach results of Grivaux [10] mentioned above continue to be hold in the setting of Fréchet spaces admitting a continuous norm. 


\section{Preliminaries}

Throughout this paper, the following notation will be used.

Let $X$ be an infinite dimensional Fréchet space over the scalar field $\mathrm{K}$, where either $\mathrm{K}=\mathrm{R}$ or $\mathrm{K}=\mathrm{C}$, and let $\left\{\|\cdot\|_{k}\right\}_{k=1}^{\infty}$ be an increasing sequence of seminorms defining the lc-topology of $X$. Then $X_{k}$ denotes the local Banach space generated by $\|\cdot\|_{k}$, i.e., $X_{k}$ is the completion of the quotient normed space $\left(X / \operatorname{Ker}\|\cdot\|_{k},\|\cdot\|_{k}\right)$. Let $\pi_{k}: X \rightarrow X_{k}$ the canonical map. Then $X=\operatorname{proj}_{k} X_{k}$ is the (reduced) projective limit of the sequences of Banach spaces $\left\{X_{k}\right\}_{k=1}^{\infty}$.

For each $k \in \mathrm{N}$, we set $U_{k}:=\left\{x \in X:\|x\|_{k} \leq 1\right\}$ (so, the set $\left\{U_{k}\right\}_{k=1}^{\infty}$ forms a basis of 0-neighbourhoods in $X$ ) and define the dual seminorm $\|\cdot\|_{k}^{\prime}$ of $\|\cdot\|_{k}$ on the topological dual $X^{\prime}$ of $X$ by

$$
\|f\|_{k}^{\prime}:=\sup \left\{|f(x)|:\|x\|_{k} \leq 1\right\}=\sup \left\{|f(x)|:\|x\|_{k}=1\right\}, \quad f \in X^{\prime},
$$

i.e, $\|\cdot\|_{k}^{\prime}$ is the gauge of the polar $\stackrel{\circ}{U}_{k}$ of $U_{k}$ in $X^{\prime}$. Let $X_{k}^{\prime}:=\left\{f \in X^{\prime}\right.$ : $\|f\|_{k}^{\prime}<\infty$ \} the linear span of $\stackrel{\circ}{U}_{k}$ endowed with the norm topology defined by $\|\cdot\|_{k}^{\prime}$. Then $\left(X_{k}^{\prime},\|\cdot\|_{k}^{\prime}\right)$ is a Banach space and the transpose map $\pi_{k}^{t}$ of the canonical map $\pi_{k}$ is an isometry from the strong dual of the Banach space $X_{k}$ (i.e., the completion of $\left.\left(X / \operatorname{Ker}\|\cdot\|_{k},\|\cdot\|_{k}\right)\right)$ onto $\left(X_{k}^{\prime},\|\cdot\|_{k}^{\prime}\right)$. Therefore, every $f \in\left(X / \operatorname{Ker}\|\cdot\|_{k},\|\cdot\|_{k}\right)^{\prime}$ defines a continuous linear functional $g=f \circ \pi_{k} \in$ $X^{\prime}$ with $\|g\|_{k}^{\prime}<\infty$. We observe that $X^{\prime}=\bigcup_{k=1}^{\infty} X_{k}^{\prime}$ holds algebraically.

The strong operator topology $\tau_{s}$ in the space $\mathscr{L}(X)$ of all continuous linear operators from $X$ into itself is determined by the family of seminorms

$$
\|S\|_{k, x}:=\|S(x)\|_{k}, \quad S \in \mathscr{L}(X),
$$

for each $x \in X$ and $k \in \mathrm{N}$, in which case we write $\mathscr{L}_{s}(X)$. Denote by $\mathscr{B}(X)$ the collection of all bounded subsets of $X$. The topology $\tau_{b}$ of uniform convergence on bounded sets is defined in $\mathscr{L}(X)$ via the seminorms

$$
\|S\|_{k, B}:=\sup _{x \in B}\|S(x)\|_{k}, \quad S \in \mathscr{L}(X),
$$

for each $B \in \mathscr{B}(X)$ and $k \in \mathrm{N}$; in this case we write $\mathscr{L}_{b}(X)$. For $(X,\|\cdot\|)$ a Banach space, $\tau_{b}$ is the operator norm topology in $\mathscr{L}(X)$ and hence, it is generated by the norm

$$
\|S\|:=\sup _{\|x\| \leq 1}\|S(x)\|, \quad S \in \mathscr{L}(X) .
$$

The identity operator on $X$ is denoted by $I$.

From now on, $X$ (always) denotes a Fréchet space which admits a continuous norm. Then we (may) assume that each $\|\cdot\|_{k}$ is a norm on $X$ and 
hence, the local Banach space $X_{k}$ is the completion of the normed space $\left(X,\|\cdot\|_{k}\right)$. For every $k \in \mathrm{N}$, the canonical map $\pi_{k}: X \rightarrow X_{k}$ is then the inclusion map and has dense range. It follows that $\pi_{k}^{t}(f)=\left.f\right|_{X}$ for all $f \in X_{k}^{\prime}$ and that $X_{k}^{\prime}$ can be identified with a $\sigma\left(X^{\prime}, X\right)$-dense linear subspace of $X^{\prime}$. Denoting by $\|\cdot\|_{k}$ the operator norm defining the lc-topology of $\mathscr{L}_{b}\left(X_{k}\right)$, i.e., $\|S\|_{k}=\sup _{x \in X_{k},\|x\|_{k} \leq 1}\|S(x)\|_{k}$ for $S \in \mathscr{L}\left(X_{k}\right)$, we observe that

$$
\|S\|_{k}=\sup _{x \in X,\|x\|_{k} \leq 1}\|S(x)\|_{k}, \quad S \in \mathscr{L}\left(X_{k}\right),
$$

because $X$ is dense in $X_{k}$. We point out that if $S \in \mathscr{L}(X)$ satisfies

$$
\|S(x)\|_{k} \leq c\|x\|_{k}, \quad x \in X,
$$

for some $k \in \mathrm{N}$ and $c>0$, then $S$ extends to a continuous linear operator on $X_{k}$, say $\bar{S}$, so that $\|\bar{S}\|_{k} \leq c$. For $y \in X$ and $f \in X^{\prime}$, the tensor product $f \otimes y$ denotes the continuous linear operator on $X$ defined by $(f \otimes y)(x)=f(x) y$ for $x \in X$. We observe that if $f \in X_{k}^{\prime}$ for some $k \in \mathbf{N}$ (hence, $\|f\|_{k}^{\prime}<\infty$ ), then we have, for each $h \in \mathrm{N}$, that

$$
\|(f \otimes y)(x)\|_{h}=|f(x)|\|y\|_{h} \leq\|f\|_{k}^{\prime}\|y\|_{h}\|x\|_{k}, \quad x \in X .
$$

Thus, $f \otimes y \in \mathscr{L}\left(X_{h}\right)$ for all $h \geq k$.

For other undefined notation and results on Fréchet spaces we refer to [15].

\section{The results}

We recall that $X$ denotes a Fréchet space (resp., a vector space) over $\mathrm{K}$, where either $\mathrm{K}=\mathrm{R}$ or $\mathrm{K}=\mathrm{C}$, and that $X^{\prime}$ (resp., $X^{*}$ ) denotes the topological dual (resp., algebraic dual) of $X$.

We begin with two lemmas, the first of which is of algebraic type and will be used to prove the second lemma.

Lemma 3.1. Let $X$ be a vector space. Let $S: X \rightarrow X$ be a linear operator and let $e \in X, e^{*} \in X^{*}$. If $S$ is invertible and $e^{*}\left(S^{-1}(e)\right) \neq-1$, then the linear operator $T: X \rightarrow X$ defined by

$$
T(x)=S(x)+e^{*}(x) e, \quad x \in X,
$$

is invertible, i.e., $T$ is bijective.

The proof of Lemma 3.1 is straightforward and hence we can skip it.

The next lemma shows that any two dense sets of linearly independent vectors of a separable, infinite dimensional Fréchet space $X$ which admits a continuous norm, are isomorphic. Hence, we extend to the setting of separable 
Fréchet spaces with a continuous norm a result due to Grivaux [10, Lemma 2.1] for separable Banach spaces. Actually, the general idea of the proof is inspired by [10, Lemma 2.1], but the proof requires dealing with new technical details of different kind because the involved space $X$ is not Banach. The main obstruction is due to the fact that the usual criterion of invertibility of operators on Banach spaces (i.e., if $\|S\|<1$ then $I-S$ is invertible with continuous inverse) no longer holds in the setting of operators on Fréchet spaces. To avoid this, we give a method to construct operators which are continuous and satisfy such a criterion in each local Banach space $X_{k}$ of the underlying Fréchet space $X$, thereby obtaining the invertibility at each step.

Lemma 3.2. Let $X$ be a separable, infinite dimensional Fréchet space which admits a continuous norm. Let $V=\left\{v_{n}: n \geq 1\right\}$ and $W=\left\{w_{n}: n \geq 1\right\}$ be two dense sets of linearly independent vectors of $X$. Then there exists a topological isomorphism $L \in \mathscr{L}(X)$ such that $L(V)=W$.

Proof. Let $\left\{\|\cdot\|_{k}\right\}_{k=1}^{\infty}$ denote an increasing sequence of norms defining the lc-topology of $X$. Let $\varepsilon \in(0,1)$ and $\left(\varepsilon_{n}\right)_{n=1}^{\infty}$ be a sequence of positive real numbers such that $\sum_{n=1}^{\infty} \varepsilon_{n}<\varepsilon$. Then there exists a sequence $\left\{L_{n}\right\}_{n \geq 0}$ of linear operators on $X$ such that $L_{0}=I$ and, for every $n \geq 1$,

(1) $L_{n} \in \mathscr{L}(X)$ and is invertible in $\mathscr{L}(X)$ (i.e., there exists $L_{n}^{-1} \in \mathscr{L}(X)$ ),

(2) $L_{n}$ extends to a continuous linear operator on $X_{k}$, denoted again by $L_{n}$ and hence $L_{n} \in \mathscr{L}\left(X_{k}\right)$, and such an extension is invertible in $\mathscr{L}\left(X_{k}\right)$ for $k \geq 1$,

(3) $\left\|L_{n}-L_{n-1}\right\|_{k}<\varepsilon_{n} /\left(\max _{h=0}^{n-1}\left\|L_{h}^{-1}\right\|_{h+1}\right)$ for $1 \leq k \leq n$,

(4) there exist two positive integers $p_{n}$ and $q_{n}$ such that $L_{n} v_{n}=w_{p_{n}}$ and $L_{n}^{-1} w_{n}=v_{q_{n}}$,

(5) $L_{n}=L_{n-1}$ on $\operatorname{span}\left\{v_{1}, \ldots, v_{n-1}, v_{q_{1}}, \ldots, v_{q_{n-1}}\right\}$ for $n \geq 2$.

The proof is given by induction. Let us begin by constructing $L_{1}$. Since $\|\cdot\|_{1}$ is a norm on $X, X_{1}^{\prime}$ is $\sigma\left(X^{\prime}, X\right)$-dense in $X^{\prime}$ and hence, there exists $x_{1}^{\prime} \in X_{1}^{\prime}$ such that $x_{1}^{\prime}\left(v_{1}\right)=1$. By the denseness of $W$ in $X$, for any $0<\alpha<1$, there is $w_{p_{1}} \in W$ such that the vector $e_{1}:=w_{p_{1}}-v_{1}$ satisfies

$$
\left\|e_{1}\right\|_{1}<\frac{\alpha}{\left\|x_{1}^{\prime}\right\|_{1}^{\prime}}
$$

We then define an operator $K_{1}: X \rightarrow X$ via

$$
K_{1}(x):=x+x_{1}^{\prime}(x) e_{1}, \quad x \in X .
$$


Since $x_{1}^{\prime} \in X_{1}^{\prime}$, we have, for every $k \geq 1$, that

$$
\begin{aligned}
\left\|K_{1}(x)\right\|_{k} & =\left\|x+x_{1}^{\prime}(x) e_{1}\right\|_{k} \leq\|x\|_{k}+\mid x_{1}^{\prime}(x)\|\| e_{1} \|_{k} \\
& \leq\|x\|_{k}+\left\|x_{1}^{\prime}\right\|_{1}^{\prime}\|x\|_{1}\left\|e_{1}\right\|_{k} \leq\left(1+\left\|x_{1}^{\prime}\right\|_{1}^{\prime}\left\|e_{1}\right\|_{k}\right)\|x\|_{k} .
\end{aligned}
$$

Therefore, $K_{1} \in \mathscr{L}(X)$ and, in particular, $K_{1}$ also extends to a continuous linear operator on $X_{k}$ for all $k \geq 1$. Denote such extension operators again by $K_{1}$ (hence, we have that $K_{1} \in \mathscr{L}\left(X_{k}\right)$ for $k \geq 1$ ).

By (2) we also have that $\left|x_{1}^{\prime}\left(e_{1}\right)\right|<\alpha$. Since $0<\alpha<1$, it follows that $0<1-\alpha<1+x_{1}^{\prime}\left(e_{1}\right)<1+\alpha$. This implies that $1+x_{1}^{\prime}\left(e_{1}\right) \neq 0$. Therefore, we can apply Lemma 3.1 and hence, the open mapping theorem to conclude that $K_{1}$ is invertible both in $\mathscr{L}(X)$ and in $\mathscr{L}\left(X_{k}\right)$ for every $k \geq 1$. Moreover, $K_{1}\left(v_{1}\right)=v_{1}+x_{1}^{\prime}\left(v_{1}\right) e_{1}=v_{1}+\left(w_{p_{1}}-v_{1}\right)=w_{p_{1}}$.

If $p_{1}=1$, we take $L_{1}:=K_{1}$. Otherwise, $w_{1}$ and $w_{p_{1}}$ are linearly independent vectors of $X$. Since $X_{1}^{\prime}$ is $\sigma\left(X^{\prime}, X\right)$-dense in $X^{\prime}$, there exists $y_{1}^{\prime} \in X_{1}^{\prime}$ such that $y_{1}^{\prime}\left(w_{p_{1}}\right)=0$ and $y_{1}^{\prime}\left(w_{1}\right)=1$. By the denseness of $V$ in $X$, for any $0<\beta<1$, there exists $v_{q_{1}} \in V$ such that the vector $f_{1}:=v_{q_{1}}-K_{1}^{-1}\left(w_{1}\right)$ satisfies

$$
\left\|f_{1}\right\|_{1}<\frac{\beta}{\left\|K_{1}\right\|_{1}\left\|y_{1}^{\prime}\right\|_{1}^{\prime}}
$$

We then define an operator $L_{1}^{-1}: X \rightarrow X$ via

$$
L_{1}^{-1}(x):=K_{1}^{-1}(x)+y_{1}^{\prime}(x) f_{1}, \quad x \in X .
$$

Since $K_{1}^{-1} \in \mathscr{L}(X)$ (resp., $K_{1}^{-1} \in \mathscr{L}\left(X_{k}\right)$ for all $k \geq 1$ ) and $y_{1}^{\prime} \in X_{1}^{\prime}$, we can proceed as above to show that $L_{1}^{-1} \in \mathscr{L}(X)$ (resp., that $L_{1}^{-1}$ extends to a continuous linear operator on $X_{k}$ for all $k \geq 1$ ).

Since $y_{1}^{\prime} \in X_{1}^{\prime}$ and $K_{1} \in \mathscr{L}\left(X_{1}\right)$, we can apply (3) to obtain that $\left|y_{1}^{\prime}\left(K_{1}\left(f_{1}\right)\right)\right|<\beta$. It follows that $0<1-\beta<1+y_{1}^{\prime}\left(K_{1}\left(f_{1}\right)\right)<1+\beta$ as $0<\beta<1$. This implies that $1+y_{1}^{\prime}\left(K_{1}\left(f_{1}\right)\right) \neq 0$. Since $K_{1}^{-1}$ is invertible both in $X$ and in $X_{k}$ for every $k \geq 1$, we can apply Lemma 3.1 and hence, the open mapping theorem, to conclude that $L_{1}^{-1}$ is invertible both in $\mathscr{L}(X)$ and in $\mathscr{L}\left(X_{k}\right)$ for every $k \geq 1$. Moreover, by construction of $K_{1}$ and of $L_{1}^{-1}$, we have that $L_{1}^{-1}\left(w_{1}\right)=K_{1}^{-1}\left(w_{1}\right)+y_{1}^{\prime}\left(w_{1}\right) f_{1}=K_{1}^{-1}\left(w_{1}\right)+f_{1}=v_{q_{1}}$ and that $L_{1}^{-1}\left(w_{p_{1}}\right)=K_{1}^{-1}\left(w_{p_{1}}\right)+y_{1}^{\prime}\left(w_{p_{1}}\right) f_{1}=K_{1}^{-1}\left(w_{p_{1}}\right)=v_{1}$. It follows that $L_{1}\left(v_{q_{1}}\right)=w_{1}$ and $L_{1}\left(v_{1}\right)=w_{p_{1}}$.

Finally, we observe that by construction either $L_{1}=K_{1}$ or $L_{1}^{-1}=K_{1}^{-1}+$ $y_{1}^{\prime} \otimes f_{1}$. If $L_{1}=K_{1}$, we clearly have $\left\|L_{1}-K_{1}\right\|_{1}=0$. Otherwise, from (3) it 
follows that

$$
\begin{gathered}
\left\|\left(L_{1}^{-1}-K_{1}^{-1}\right)(x)\right\|_{1}=\left|y_{1}^{\prime}(x)\right|\left\|f_{1}\right\|_{1} \leq\left\|y_{1}^{\prime}\right\|_{1}^{\prime}\|x\|_{1}\left\|f_{1}\right\|_{1}<\frac{\beta}{\left\|K_{1}\right\|_{1}}\|x\|_{1}, \\
x \in X_{1} \text {, i.e., }\left\|L_{1}^{-1}-K_{1}^{-1}\right\|_{1}<\frac{\beta}{\left\|K_{1}\right\|_{1}} \text {. Consequently, we obtain } \\
\left\|L_{1}-K_{1}\right\|_{1}=\left\|L_{1} K_{1}^{-1} K_{1}-L_{1} L_{1}^{-1} K_{1}\right\|_{1}=\left\|L_{1}\left(K_{1}^{-1}-L_{1}^{-1}\right) K_{1}\right\|_{1} \\
\leq\left\|L_{1}\right\|_{1}\left\|L_{1}^{-1}-K_{1}^{-1}\right\|_{1}\left\|K_{1}\right\|_{1}<\beta\left\|L_{1}\right\|_{1} .
\end{gathered}
$$

Then, in either case, by (2) we have that

$$
\begin{aligned}
\left\|L_{1}-L_{0}\right\|_{1} & =\left\|L_{1}-L_{0}-x_{1}^{\prime} \otimes e_{1}+x_{1}^{\prime} \otimes e_{1}\right\|_{1}=\left\|\left(L_{1}-K_{1}\right)+x_{1}^{\prime} \otimes e_{1}\right\|_{1} \\
& \leq\left\|L_{1}-K_{1}\right\|_{1}+\left\|x_{1}^{\prime} \otimes e_{1}\right\|_{1}<\beta\left\|L_{1}\right\|_{1}+\alpha<\varepsilon_{1}=\frac{\varepsilon_{1}}{\left\|L_{0}^{-1}\right\|_{1}}
\end{aligned}
$$

if $\alpha$ and $\beta$ are small enough to satisfy the condition $\beta\left\|L_{1}\right\|_{1}+\alpha<\varepsilon_{1}$ (here, $\left.\left\|L_{0}^{-1}\right\|_{1}=1\right)$.

Suppose that $L_{1}, \ldots, L_{n}$ have already been constructed in such a way that all properties (1)-(5) are satisfied.

If $n+1 \in\left\{q_{1}, \ldots, q_{n}\right\}$, we take $K_{n+1}:=L_{n}$. Otherwise, $v_{n+1}$ does not belong to the vector space $\operatorname{span}\left\{v_{1}, \ldots, v_{n}, v_{q_{1}}, \ldots, v_{q_{n}}\right\}$ because the vectors $v_{i}$ are linearly independent. Since $X_{1}^{\prime}$ is $\sigma\left(X^{\prime}, X\right)$-dense in $X^{\prime}$, we can find $x_{n+1}^{\prime} \in$ $X_{1}^{\prime}$ such that $x_{n+1}^{\prime}\left(v_{n+1}\right)=1$ and $x_{n+1}^{\prime}\left(v_{i}\right)=0$ for $i \in\left\{1, \ldots, n, q_{1}, \ldots, q_{n}\right\}$. By the denseness of $W$ in $X$, for any $0<\alpha<1$, there is $w_{p_{n+1}}$ in $W$ so that the vector $e_{n+1}:=w_{p_{n+1}}-L_{n}\left(v_{n+1}\right)$ satisfies

$$
\left\|e_{n+1}\right\|_{n+1}<\frac{\alpha}{\max _{h=0}^{n}\left\|L_{h}^{-1}\right\|_{h+1}} \frac{1}{\left\|x_{n+1}^{\prime}\right\|_{1}^{\prime}} .
$$

We then define an operator $K_{n+1}: X \rightarrow X$ by

$$
K_{n+1}(x):=L_{n}(x)+x_{n+1}^{\prime}(x) e_{n+1}, \quad x \in X .
$$

Using properties (1) and (2) of the inductive step and the fact that $x_{n+1}^{\prime} \in X_{1}^{\prime}$, we can proceed as above to show that $K_{n+1} \in \mathscr{L}(X)$ (resp., that $K_{n+1}$ extends to a continuous linear operator on $X_{k}$ for all $k \geq 1$ ).

Since $x_{n+1}^{\prime} \in X_{1}^{\prime} \subset X_{n+1}^{\prime},\left\|x_{n+1}^{\prime}\right\|_{n+1}^{\prime} \leq\left\|x_{n+1}^{\prime}\right\|_{1}^{\prime}$ and $L_{n}^{-1} \in \mathscr{L}\left(X_{n+1}\right)$, the property (3) of inductive step together with (4) imply that $\left|x_{n+1}^{\prime}\left(L_{n}^{-1}\left(e_{n+1}\right)\right)\right|<$ $\alpha$. It follows that $0<1-\alpha<1+x_{n+1}^{\prime}\left(L_{n}^{-1}\left(e_{n+1}\right)\right)<1+\alpha$ as $0<\alpha<1$. This implies that $1+x_{n+1}^{\prime}\left(L_{n}^{-1}\left(e_{n+1}\right)\right) \neq 0$. Since by properties (1) and (2) of the inductive step $L_{n}$ is invertible both in $\mathscr{L}(X)$ and in $\mathscr{L}\left(X_{k}\right)$ for every $k \geq 1$, we can apply again Lemma 3.1 together with the open mapping theorem to 
conclude that $L_{n+1}$ is invertible both in $\mathscr{L}(X)$ and in $\mathscr{L}\left(X_{k}\right)$ for every $k \geq 1$. Moreover, by construction $K_{n+1}\left(v_{n+1}\right)=w_{p_{n+1}}$ and, by properties (4) and (5) of the inductive step, $K_{n+1}\left(v_{k}\right)=L_{k}\left(v_{k}\right)=w_{p_{k}}$ and that $K_{n+1}\left(v_{q_{k}}\right)=$ $L_{k}\left(v_{q_{k}}\right)=w_{k}$ for every $1 \leq k \leq n$.

Let us construct $L_{n+1}$. If $n+1 \in\left\{p_{1}, \ldots, p_{n+1}\right\}$, we take $L_{n+1}:=K_{n+1}$. Otherwise, $w_{n+1}$ does not belong to the vector space $\operatorname{span}\left\{w_{p_{1}}, \ldots, w_{p_{n+1}}\right.$, $w_{1}, \ldots, w_{n}$ \} because the vectors $w_{j}$ are linearly independent. Since $X_{1}^{\prime}$ is $\sigma\left(X^{\prime}, X\right)$ dense in $X^{\prime}$, we can find $y_{n+1}^{\prime} \in X_{1}^{\prime}$ such that $y_{n+1}^{\prime}\left(w_{n+1}\right)=1$ and $y_{n+1}^{\prime}\left(w_{i}\right)=0$ if $i \in\left\{1, \ldots, n, p_{1}, \ldots, p_{n+1}\right\}$. By the denseness of $V$ in $X$, for any $0<\beta<1$, there exists an element $v_{q_{n+1}}$ in $V$ for which the vector $f_{n+1}:=v_{q_{n+1}}-K_{n+1}^{-1}\left(w_{n+1}\right)$ satisfies

$$
\left\|f_{n+1}\right\|_{n+1}<\frac{\beta}{\max _{h=1}^{n+1}\left\|K_{n+1}\right\|_{h}} \frac{1}{\left\|y_{n+1}^{\prime}\right\|_{1}^{\prime}} .
$$

We then define an operator $L_{n+1}^{-1}: X \rightarrow X$ via

$$
L_{n+1}^{-1}(x):=K_{n+1}^{-1}(x)+y_{n+1}^{\prime}(x) f_{n+1}, \quad x \in X .
$$

Since $K_{n+1}^{-1} \in \mathscr{L}(X)$ (resp., $K_{n+1}^{-1} \in \mathscr{L}\left(X_{k}\right)$ for all $k \geq 1$ ) and $y_{n+1}^{\prime} \in X_{1}^{\prime}$, we can proceed as in the first step to show that $L_{n+1}^{-1} \in \mathscr{L}(X)$ (resp., that $L_{n+1}^{-1}$ extends to a continuous linear operator on $X_{k}$ for all $k \geq 1$ ).

Since $y_{n+1}^{\prime} \in X_{1}^{\prime} \subset X_{n+1}^{\prime},\left\|y_{n+1}^{\prime}\right\|_{n+1}^{\prime} \leq\left\|y_{n+1}^{\prime}\right\|_{1}^{\prime}$ and $K_{n+1} \in \mathscr{L}\left(X_{n+1}\right)$, inequality (5) implies that $\left|y_{n+1}^{\prime}\left(K_{n+1}\left(f_{n+1}\right)\right)\right|<\beta$. It follows that $0<$ $1-\beta<1+y_{n+1}^{\prime}\left(K_{n+1}\left(f_{n+1}\right)\right)<1+\beta$ as $0<\beta<1$. This implies that $1+y_{n+1}^{\prime}\left(K_{n+1}\left(f_{n+1}\right)\right) \neq 0$. Since $K_{n+1}^{-1}$ is invertible both in $X$ and in $X_{k}$ for every $k \geq 1$, we can apply Lemma 3.1 and hence, the open mapping theorem, to conclude that $L_{n+1}^{-1}$ is invertible both in $\mathscr{L}(X)$ and in $\mathscr{L}\left(X_{k}\right)$ for every $k \geq 1$. Moreover, by construction of $K_{n+1}$ and of $L_{n+1}^{-1}$, we have that $L_{n+1}^{-1}\left(w_{n+1}\right)=v_{q_{n+1}}$ and that the operators $K_{n+1}^{-1}$ and $L_{n+1}^{-1}$ coincide on $\operatorname{span}\left\{w_{p_{1}}, \ldots, w_{p_{n+1}}, w_{1}, \ldots, w_{n}\right\}$. Thus, for every $1 \leq k \leq n+1$, $L_{n+1}^{-1}\left(w_{p_{k}}\right)=K_{n+1}^{-1}\left(w_{p_{k}}\right)=v_{k}$ so that $L_{n+1}\left(v_{k}\right)=w_{p_{k}}$. In particular, $L_{n+1}=$ $L_{n}$ on $\operatorname{span}\left\{v_{1}, \ldots, v_{n}\right\}$. We also have, for every $1 \leq k \leq n$, that $L_{n+1}^{-1}\left(w_{k}\right)=$ $K_{n+1}^{-1}\left(w_{k}\right)=v_{q_{k}}$ so that $L_{n+1}\left(v_{q_{k}}\right)=w_{k}$ and $L_{n+1}=L_{n}$ on $\operatorname{span}\left\{v_{q_{1}}, \ldots, v_{q_{n}}\right\}$. We have so shown that $L_{n+1}$ satisfies properties (1), (2), (4) and (5).

Finally, we observe that by construction either $L_{n+1}=K_{n+1}$ or $L_{n+1}^{-1}=$ $K_{n+1}^{-1}+y_{n+1}^{\prime} \otimes f_{n+1}$. If $L_{n+1}=K_{n+1}$, we clearly have, for $1 \leq k \leq n+1$, that $\left\|L_{n+1}-K_{n+1}\right\|_{k}=0$. Otherwise, using (5) and proceeding as above, one shows that $\left\|L_{n+1}^{-1}-K_{n+1}^{-1}\right\|_{k}<\frac{\beta}{\max _{h=1}^{n+1}\left\|K_{n+1}\right\|_{h}}$ for every $1 \leq k \leq n+1$. Consequently, we obtain that $\left\|L_{n+1}-K_{n+1}\right\|_{k} \leq \beta\left\|L_{n+1}\right\|_{k}$ for every $1 \leq k \leq n+1$. Then, 
in either case, by (4) we obtain as in the first step, for $1 \leq k \leq n+1$, that

$$
\left\|L_{n+1}-L_{n}\right\|_{k}<\frac{\varepsilon_{n+1}}{\max _{h=0}^{n}\left\|L_{h}^{-1}\right\|_{h+1}},
$$

if $\alpha$ and $\beta$ are enough small to have $\beta\left\|L_{n+1}\right\|_{k}+\frac{\alpha}{\max _{h=0}^{n}\left\|L_{h}^{-1}\right\|_{h+1}}<\frac{\varepsilon_{n+1}}{\max _{h=0}^{n}\left\|L_{h}^{-1}\right\|_{h+1}}$. So, $L_{n+1}$ also satisfies property (3). This completes the induction proof.

Now, we set $L:=I+\sum_{n=0}^{\infty}\left(L_{n+1}-L_{n}\right)$. Then, the operator $L$ is well defined in $X$ (resp., in $X_{k}$ for $k \geq 1$ ) and belongs to $\left.\mathscr{L}(X)\right)$ (resp., to $\mathscr{L}\left(X_{k}\right)$ for $k \geq 1$ ). Indeed, for a given $k \in \mathrm{N}$, by property (3) we have that $\left\|L_{n+1}-L_{n}\right\|_{k}<$ $\varepsilon_{n+1}$ for every $n \geq k-1$. Since $\sum_{n \geq k-1} \varepsilon_{n+1}<\varepsilon$, it follows that the series $\sum_{n=0}^{\infty}\left\|L_{n+1}-L_{n}\right\|_{k}$ converges. Therefore, since $k$ is arbitrary, we can conclude that $L$ is well defined both in $X$ and in each $X_{k}$. Moreover, by property (2) we have, for every $k \in \mathrm{N}$, that

$$
\begin{aligned}
\|L(x)\|_{k} & \leq\|x\|_{k}+\left\|\sum_{n=0}^{k-2}\left(L_{n+1}-L_{n}\right)(x)\right\|_{k}+\sum_{n=k-1}^{\infty}\left\|\left(L_{n+1}-L_{n}\right)(x)\right\|_{k} \\
& \leq\left(1+c_{k}+\varepsilon\right)\|x\|_{k},
\end{aligned}
$$

$x \in X_{k}$, with $c_{k}$ a suitable positive constant. Since $X \subseteq X_{k}$, this ensures that $L \in \mathscr{L}(X)$ and that $L \in \mathscr{L}\left(X_{k}\right)$ for every $k \geq 1$. Moreover, $L$ is invertible in each $\mathscr{L}\left(X_{k}\right)$. Indeed, fix any $k \in \mathrm{N}$. Then, by property (2) we can write

(6) $L=L_{k-1}+\sum_{n=k-1}^{\infty}\left(L_{n+1}-L_{n}\right)=L_{k-1}\left[I+L_{k-1}^{-1} \sum_{n=k-1}^{\infty}\left(L_{n+1}-L_{n}\right)\right]$

in $X_{k}$. On the other hand, by property (3), we have, for every $n \geq k-1$, that

$$
\begin{aligned}
\left\|L_{k-1}^{-1}\left(L_{n+1}-L_{n}\right)\right\|_{k} & \leq\left\|L_{k-1}^{-1}\right\|_{k}\left\|L_{n+1}-L_{n}\right\|_{k} \\
& \leq\left\|L_{k-1}^{-1}\right\|_{k} \frac{\varepsilon_{n+1}}{\max _{h=0}^{n}\left\|L_{h}^{-1}\right\|_{h+1}} \leq \varepsilon_{n+1} .
\end{aligned}
$$

Since the series $\sum_{n=k-1}^{\infty}\left(L_{n+1}-L_{n}\right)$ converges in $\mathscr{L}_{b}\left(X_{k}\right)$ and $L_{k-1}^{-1} \in \mathscr{L}\left(X_{k}\right)$, from (7) it follows

$$
\begin{aligned}
\left\|L_{k-1}^{-1} \sum_{n=k-1}^{\infty}\left(L_{n+1}-L_{n}\right)\right\|_{k} & =\left\|\sum_{n=k-1}^{\infty} L_{k-1}^{-1}\left(L_{n+1}-L_{n}\right)\right\|_{k} \\
& \leq \sum_{j=k}^{\infty} \varepsilon_{j}<\varepsilon<1 .
\end{aligned}
$$


Combining (6) with (8) and property (2), we can conclude that the operator $L$ is invertible in $\mathscr{L}\left(X_{k}\right)$. Therefore, there exist two positive constants $a_{k}$ and $b_{k}$ such that

$$
a_{k}\|x\|_{k} \leq\|L x\|_{k} \leq b_{k}\|x\|_{k}, \quad x \in X_{k} .
$$

Since $X \subseteq X_{k}$ and $k$ is arbitrary, from (9) it follows that $L$ is an injective continuous and open linear operator from $X$ into $X$. Moreover, in view of properties (4) and (5), we can argument as in [10, Lemma 2.1] to conclude that $L(V)=W$. As $V$ and $W$ are dense subsets of $X$, this equality together with (9) imply that the operator $L$ is also surjective. Therefore, $L$ is invertible in $\mathscr{L}(X)$, i.e., $L$ is a topological isomorphism of $X$.

REMARK 3.3. The topological isomorphisms constructed in Lemma 3.2 are of the form $L=I+K$ with $K$ a nuclear operator on each $X_{k}$ (hence, $K$ is a nuclear operator on $X$ ). Actually, by a slight modification in the proof of Lemma 3.2 we can show that, for every $h \in \mathrm{N}$ there exists a topological isomorphism $L \in \mathscr{L}(X)$ of the form $L=I+K$ such that $L(V)=W$, $\|L-I\|_{h}<\varepsilon$ and $\left\|L^{-1}-I\right\|_{h}<\frac{\varepsilon}{1-\varepsilon}$.

Remark 3.4. Let $X$ be a separable, infinite dimensional Fréchet space which admits a continuous norm. If $V=\left\{v_{n}: n \geq 1\right\}$ and $W=\left\{w_{n}\right.$ : $n \geq 1\}$ are two dense sets of linearly independent vectors in $X$, then for every $m \in \mathrm{N}$ there exists a topological isomorphism $L$ on $X$ such that $L(V)=$ $W$ and $L\left(v_{i}\right)=w_{i}$ for $1 \leq i \leq m$. Indeed, by Lemma 3.2 there exists a topological isomorphism $L_{0}=I+K_{0}$ on $X$, with $K_{0}$ a nuclear operator, such that $L_{0}(V)=W$. Then, for every $1 \leq i \leq m, L_{0}\left(v_{i}\right)=w_{p_{i}}$ for some $p_{i} \in \mathrm{N}$, where by construction $p_{i} \neq p_{j}$ if $i \neq j$. Without of loss of generality, we may suppose that $p_{i} \neq i$ for $1 \leq i \leq m$ (eventually, by deleting the indeces $i$ for which $p_{i}=i$ ). Therefore, the vectors $w_{1}, \ldots, w_{m}, w_{p_{1}}, \ldots w_{p_{m}}$ are linearly independent and hence, since $X_{1}^{\prime}$ is $\sigma\left(X^{\prime}, X\right)$ dense in $X^{\prime}$, there exist $y_{1}^{\prime}, \ldots, y_{m}^{\prime}, y_{p_{1}}^{\prime}, \ldots, y_{p_{m}}^{\prime} \in X_{1}^{\prime}$ such that $y_{j}^{\prime}\left(w_{i}\right)=\delta_{i j}, y_{j}^{\prime}\left(w_{p_{i}}\right)=0$, $y_{p_{j}}^{\prime}\left(w_{i}\right)=0$ and, $y_{p_{j}}^{\prime}\left(w_{p_{i}}\right)=\delta_{i j}$ for $i, j=1, \ldots, m$. We then define a continuous linear projection $P$ on $X$ by setting

$$
P(x)=\sum_{i=1}^{m} y_{i}^{\prime}(x) w_{i}+\sum_{i=1}^{m} y_{p_{i}}^{\prime}(x) w_{p_{i}}, \quad x \in X .
$$

Hence, $\operatorname{Im} P=\operatorname{span}\left\{w_{1}, \ldots, w_{m}, w_{p_{1}}, \ldots w_{p_{m}}\right\}$ and $X=\operatorname{Ker} P \oplus \operatorname{Im} P$. We point out that $P$ is also (extends to) a continuous linear projection $P$ on each $X_{k}$ because $y_{1}^{\prime}, \ldots, y_{m}^{\prime}, y_{p_{1}}^{\prime}, \ldots, y_{p_{m}}^{\prime}\left(\right.$ resp., $\left.w_{1}, \ldots, w_{m}, w_{p_{1}}, \ldots w_{p_{m}}\right)$ belong to $X_{1}^{\prime}$ (resp., $X$ ) and hence, to $X_{k}^{\prime}$ (resp., $X_{k}$ ). 
Next, we consider an operator $L_{1}: X \rightarrow X$ defined by

$$
L_{1}(x)=(I-P)(x)+\sum_{i=1}^{m} y_{i}^{\prime}(x) w_{p_{i}}+\sum_{i=1}^{m} y_{p_{i}}^{\prime}(x) w_{i}, \quad x \in X .
$$

Since $X=\operatorname{Ker} P \oplus \operatorname{Im} P$ and $\operatorname{Im} P=\operatorname{span}\left\{w_{1}, \ldots, w_{m}, w_{p_{1}}, \ldots w_{p_{m}}\right\}, L_{1}$ is a topological isomorphism on $X$ (resp., on $X_{k}$ for $k \geq 1$ ). Moreover, $L\left(w_{i}\right)=$ $w_{p_{i}}$ and $L\left(w_{p_{i}}\right)=w_{i}$ for $i=1, \ldots, m$. Then $L:=L_{1} L_{0}$ is a topological isomorphism on $X$ (resp., on $X_{k}$ for $k \geq 1$ ) such that, for $i=1, \ldots, m$, $L\left(v_{i}\right)=L_{1}\left(L_{0}\left(v_{i}\right)\right)=L_{1}\left(w_{p_{i}}\right)=w_{i}$. Finally, we observe that

$$
L_{1}-I=\sum_{i=1}^{m}\left(w_{i}-w_{p_{i}}\right) \otimes\left(y_{p_{i}}^{\prime}-y_{i}^{\prime}\right)
$$

and hence, $L_{1}-I$ is a nuclear operator on $X$ (resp., on $X_{k}$ for $k \geq 1$ ). Since we can write $L=L_{1} L_{0}=I+L_{1}\left[L_{1}^{-1}\left(L_{1}-I\right)+K_{0}\right]$, it follows that $L$ has the same form of $L_{0}$, i.e., $L=I+K$, with $K$ a nuclear operator on $X$ (resp., on $X_{k}$ for $k \geq 1$.

We observe that Lemma 3.2 shows that "any two dense subspaces of countable algebraic dimension of a separable Fréchet space $X$ which admits a continuous norm, are isomorphic", thus obtaining an extension of a result which is well-known for separable Hilbert spaces and was shown to be also valid in separable Banach spaces by Grivaux [10, Lemma 2.1]. In contrast to Lemma 3.2 and [10, Lemma 2.1], Bonet, Frerick, Peris and Wengenroth have shown in [4] that every countable product of copies of an infinite dimensional Banach space $X$ contains two dense linearly independent sequences of vectors such that their spans are not isomorphic.

We can now state and show the main result of this paper.

Theorem 3.5. Let $X$ be a separable, infinite dimensional Fréchet space which admits a continuous norm. Let $V=\left\{v_{n}: n \geq 1\right\}$ be a dense set of linearly independent vectors of $X$. Then there exists an operator $T \in \mathscr{L}(X)$ of the form $T=I+K$, with $K$ a nuclear operator on $X$, such that the orbit of $v_{1}$ under $T$ is exactly the set $\left\{v_{n}: n \geq 1\right\}$.

Proof. By the existence theorem of [6, Theorem 1] (see, also [1], [2]) there exists a hypercyclic surjective operator on $X$. Since $X$ admits a continuous norm and hence, $X \not \omega$, the hypercyclic surjective operators constructed in [6, Lemma 3] are of the form $I+K$, with $K$ a nuclear operator on $X$.

Let $T_{0}=I+K_{0}$ be a hypercyclic surjective operator on $X$ and $x_{0}$ be a hypercyclic vector for $T_{0}$. Then the orbit of $x_{0}$ under $T_{0}$, i.e., $W:=\left\{T_{0}^{n}\left(x_{0}\right) \mid\right.$ 
$n \geq 0\}$, is a dense set of linearly independent vectors of $X$. We can now apply Lemma 3.2 and Remark 3.4 to the sets $V$ and $W$ to conclude that there exists a topological isomorphism $L$ on $X$ such that $L\left(v_{1}\right)=x_{0}$ and $L(V)=W$. Next, we consider the operator $T=L^{-1} T_{0} L$. So, $T \in \mathscr{L}(X)$ and $T^{n}\left(v_{1}\right)=$ $L^{-1} T_{0}^{n}\left(x_{0}\right)$ for every $n \geq 0$. Thus, the orbit of $v_{1}$ under $T$ is the set $L^{-1}(W)$, i.e., the set $V$. Finally, we observe that $T$ is of the form $T=I+K$, where $K=L^{-1} K_{0} L$ and hence, $K$ is a nuclear operator on $X$.

We end the paper by collecting some consequences of Theorem 3.5 along the lines of [10]. Their proofs are also inspired by [10] and based on the results obtained above.

Corollary 3.6. Let $X$ be a separable, infinite dimensional Fréchet space which admits a continuous norm. Let $M$ be a dense, infinite dimensional subspace of $X$ of countable algebraic dimension. For every non-zero vector $x$ in $M$, there exists an operator $T \in \mathscr{L}(X)$ such that $M=\mathrm{K}[T](x)$.

Proof. Fix any non-zero $x \in M$. Let $V=\left\{v_{n}: n \geq 1\right\}$ be a dense algebraic basis of $M$ with $v_{1}=x$. Then we apply Theorem 3.5 to the set $V$ to exhibit an operator $T \in \mathscr{L}(X)$ such that the orbit of $v_{1}$ under $T$ is exactly the set $V$. Hence, $M$ is exactly equal to $\mathrm{K}[T]\left(v_{1}\right)$ as $V$ is an algebraic basis of $M$.

COROLlary 3.7. Let $M$ be an infinite dimensional metrizable locally convex space of countable algebraic dimension. If the completion of $M$ is a Fréchet space $X$ which admits a continuous norm, then there exists an operator $T \in$ $\mathscr{L}(M)$ such that every non-zero vector of $M$ is hypercyclic for $T$, i.e., $T$ has no non-trivial invariant closed set.

Proof. Since $X$ is a separable, infinite dimensional Fréchet space which admits a continuous norm, we can consider the operator $T \in \mathscr{L}(X)$ obtained in Corollary 3.6. Then the space $M$ is invariant under $T$. Moreover, the orbit of $v_{1}$ under $T$ is dense in $M$ and hence, in $X$. The operator $T$ is then hypercyclic and satisfies $M=\mathrm{K}[T]\left(v_{1}\right)$. This implies that every non-zero vector of $M$ is hypercyclic for $T$, see [3], [7], [14]. So, to complete the proof it suffices to consider the restriction of $T$ to $M$, i.e., $\left.T\right|_{M}$.

REMARK 3.8. We point out that Corollary 3.7 no longer holds in general locally convex spaces of countable algebraic dimension. Indeed, in [4, Proposition 3.2(b)] it is shown that there exists a countable dimensional locally convex space admitting no transitive operator and hence, no hypercyclic operators.

We denote by $\mathscr{H C}(X)$ the set of all hypercyclic operators on $X$ and by $\overline{\mathscr{H C} C}(X)$ the closure of $\mathscr{H C C}(X)$ in $\mathscr{L}_{b}(X)$. The set $\mathscr{H} \mathscr{C}(X)$ is always non-void, see [1], [2], [6]. For a given linear subspace $M$ of $X$, we denote by $\mathscr{H} C_{M}(X)$ 
the set of operators $S$ on $X$ such that $M$ is a hypercyclic linear subspace for $S$. Then we have

Corollary 3.9. Let $X$ be a separable, infinite dimensional Fréchet space which admits a continuous norm. Let $M$ be a linear subspace of $X$ of countable algebraic dimension. Then the set $\mathscr{H C}_{M}(X)$ is dense in $\overline{\mathscr{H C}}(X)$ with respect to the lc-topology of $\mathscr{L}_{b}(X)$.

Proof. We observe that it suffices to show that the set $\mathscr{H C}_{M}(X)$ is dense in $\mathscr{H C}(X)$ with respect to the lc-topology of $\mathscr{L}_{b}(X)$, i.e., that for every $T_{0} \in$ $\mathscr{H} \mathscr{C}(X), \varepsilon>0, k \in \mathrm{N}$ and $B \in \mathscr{B}(X)$ there exists $T \in \mathscr{H C C}_{M}(X)$ such that $\left\|T-T_{0}\right\|_{k, B}=\sup _{x \in B}\left\|\left(T-T_{0}\right)(x)\right\|_{h}<\varepsilon$.

Fix $T_{0} \in \mathscr{H} \mathscr{C}(X), \varepsilon>0, k \in \mathrm{N}$ and $B \in \mathscr{B}(X)$. Then the operator $T_{0}$ is hypercyclic and hence, there exists a dense hypercyclic linear subspace $V$ for $T_{0}$, see [3], [7], [14]. Since $T_{0} \in \mathscr{L}(X)$, there exist also $h \geq k$ and $c>0$ such that $\left\|T_{0}(x)\right\|_{k} \leq c\|x\|_{h}$ for all $x \in X$. Moreover, by Lemma 3.2 and Remark 3.3 for any $0<\alpha<1$ there exists a topological isomorphism $L$ on $X$ such that $L(M) \subseteq V$ and $\|L-I\|_{h}<\alpha,\left\|L^{-1}-I\right\|_{h}<\frac{\alpha}{1-\alpha}$. Then $T=L^{-1} T_{0} L \in$ $\mathscr{H C}_{M}(X)$ and $\left\|\left(T-T_{0}\right)(x)\right\|_{k} \leq c d \alpha\left\|L^{-1}\right\|_{k}+d^{\prime} \frac{\alpha}{1-\alpha}$ for every $x \in B$, with $d=\sup _{x \in B}\|x\|_{h}<\infty$ and $d^{\prime}=\sup _{x \in B}\left\|T_{0}(x)\right\|_{h}<\infty$ as $B \in \mathscr{B}(X)$ and $T_{0} \in \mathscr{L}(X)$. If $\alpha$ is small enough, it follows that $\sup _{x \in B}\left\|\left(T-T_{0}\right)(x)\right\|_{k}<\varepsilon$ and the proof is complete.

\section{REFERENCES}

1. Ansari, S. I., Existence of hypercyclic operators on topological vector spaces, J. Funct. Anal. 148 (1997), 384-390.

2. Bernal-González, L., On hypercyclic operators on Banach spaces, Proc. Amer. Math. Soc. 127 (1999), 1003-1010.

3. Bès, J., Invariant manifolds of hypercyclic vectors for the real scalar case, Proc. Amer. Math. Soc. 127 (1999), 1801-1804.

4. Bonet, J., Frerick, L., Peris, A., Wengenroth, J., Transitive and hypercyclic operators on locally convex spaces, Bull. London Math. Soc. 37 (2005), 254-264.

5. Bonet, J., Martínez-Giménez, F., Peris, A., Linear chaos on Fréchet spaces, Internat. J. Bifur. Chaos Appl. Sci. Engrg. 13 (2003), 1649-1655.

6. Bonet, J., Peris, A., Hypercyclic operators on non-normable Fréchet spaces, J. Funct. Anal. 159 (1998), 587-595.

7. P. Bourdon, Invariant manifolds of hypercyclic vectors, Proc. Amer. Math. Soc. 118 (1993), 845-847.

8. Gethner, R. M., Shapiro, J. H., Universal vectors for operators on spaces of holomorphic functions, Proc. Amer. Math. Soc. 100 (1987), 281-288.

9. Godefroy, G., Shapiro, J. H., Operators with dense, invariant, cyclic vector manifolds, J. Funct. Anal. 98 (1991), 229-269.

10. Grivaux, S., Construction of operators with prescribed behaviour, Arch. Math. (Basel) 81 (2003), 291-299. 
11. Grosse-Erdmann, K. G., Universal families and hypercyclic operators, Bull. Amer. Math. Soc. (N.S.) 36 (1999), 345-381.

12. Grosse-Erdmann, K. G., Recent developments in hypercyclicity, RACSAM Rev. R. Acad. Cienc. Exactas (A) 97 (2003), 273-286.

13. Halperin, I., Kitai, C., Rosenthal, P., On orbits of linear operators, J. London Math. Soc. (2) 31 (1985), 561-565.

14. Herrero, D. A., Limits of hypercyclic and supercyclic operators, J. Funct. Anal. 99 (1991), 179-190.

15. Meise, R., Vogt, D., Introduction to Functional Analysis, Oxford Grad. Texts in Math. 2, Clarendon Press, Oxford 1997.

16. Metafune, G., Moscatelli, V. B., Dense subspaces with continuous norm in Fréchet spaces, Bull. Polish Acad. Sci. Math. 37 (1989), 477-479.

17. Rolewicz, S., On orbits of elements, Studia Math. 32 (1969), 17-22.

18. Salas, H., Hypercyclic weighted shifts, Trans. Amer. Math. Soc. 347 (1995), 993-1004.

19. Wengenroth, J., Hypercyclic operators on non-locally convex spaces, Proc. Amer. Math. Soc. 131 (2003), 1759-1761.

DIPARTIMENTO DI MATEMATICA "E. DE GIORGI"

UNIVERSITÀ DEL SALENTO

VIA PROVINCIALE PER ARNESANO

P.O. BOX 193

I-73100 LECCE

ITALY

E-mail: angela.albanese@unile.it 